\title{
Spatial Resolving SiPM-Detector for Cosmic Muons
}

\author{
Alexander RUSCHKE ${ }^{* \dagger}$ \\ LMU-Munich \\ E-mail: alexander.ruschkeephysik.uni-muenchen. de
}

\section{Otmar Biebel}

LMU-Munich

E-mail: otmar.biebelephysik. uni-muenchen.de

\section{Johannes Grossmann}

LMU-Munich

E-mail: johannes.grossmann@physik.uni-muenchen.de

\section{Ralf Hertenberger}

LMU-Munich

E-mail: R.Hertenberger@physik . uni-muenchen. de

\section{Ralph Müller}

LMU-Munich

E-mail: ralph.mueller@physik.uni-muenchen.de

A scintillation detector with two-dimensional position resolution of a few mm and Silicon-PhotoMultiplier (SiPM)-readout is presented, usable for large area trigger applications with few electronic channels. Position resolution in one direction is achieved by combining two trapezoidal shaped plastic scintillator rods to form one rectangular shaped module. Both trapezoids are optically insulated against each other. The amount of light produced by incoming particles is proportional to the path length in the trapezoids and thus position dependent. The position resolution along the scintillator rods is determined by the propagation time of light. In both trapezoids the scintillation light is collected by Wavelength Shifting (WLS)-fibers and guided to SiPMs, where the light output is detected. The SiPMs are located on opposite sides of the scintillator rods. This detector is designed for large area applications with only few readout channels, whereby areas of square meter in size can be achieved by combining several detector modules. We present results of a test-experiment with a $120 \mathrm{GeV} \pi$-beam at CERN and report on the performance of the prototype detector.

The European Physical Society Conference on High Energy Physics

18-24 July, 2013

Stockholm, Sweden

\footnotetext{
* Speaker.

$\dagger$ The author acknowledges the support by the DFG-Graduiertenkolleg "Particle Physics at the Energy frontier to new phenomena"
} 


\section{Introduction}

The concept of the position sensitive scintillating detector is based on two trapezoidal shaped plastic scintillators (BC-400) [1], which are coupled to form a rectangular detector module, see figure 1. The detector halves are optically insulated from each other by a reflective cover material. The scintillation light from ionizing particles is collected by Wavelength-Shifting (WLS)-fibers (BCF 92) [1] and guided to Silicon-Photo-Multipliers (SiPMs), where the incoming photons are detected.

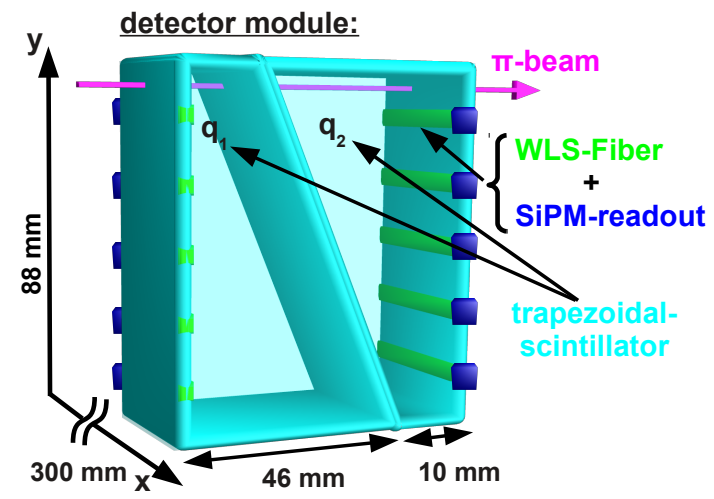

Figure 1: Sketch of the position sensitive scintillating detector. The trapezoids of the prototype detector tested at CERN were equipped with five WLS-Fibers, five SiPMs and were wrapped in aluminum foil.

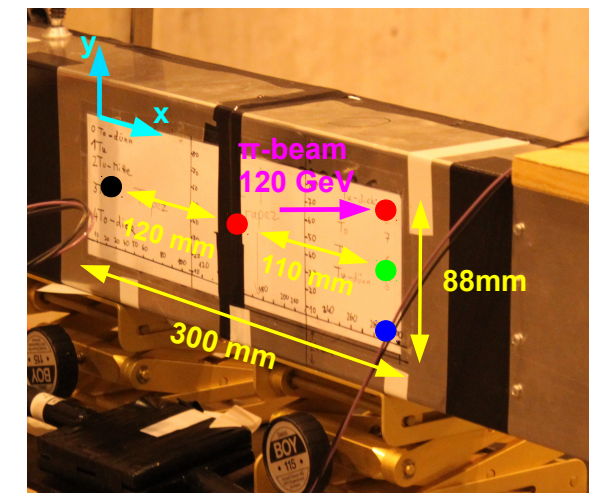

Figure 2: Light tight box with temperature monitoring at the experimental site. The white area indicates the position sensitive area of the trapezoidal detector.

\subsection{Geometry based position resolution}

The basic idea is to measure the light yield of minimum ionizing particles in each trapezoid separately. Because of the geometrical shape, the amount of scintillation light created by transversing particles is position dependent, as indicated in figure 1, and therefore a prediction of the particle position in the y-coordinate can be made by using the light yield ratio of both scintillators.

$$
\text { position prediction in } \mathrm{y} \propto \frac{q_{1}}{\left(q_{1}+q_{2}\right)}, \text { where }\left\{\begin{array}{l}
q_{1}=\text { light yield in trapezoid } 1 \\
q_{2}=\text { light yield in trapezoid } 2
\end{array}\right.
$$

The sum of $q_{1}$ and $q_{2}$ is approximately constant for perpendicular incidence.

\subsection{Position resolution by the propagation time of light}

The position resolution along the $\mathrm{x}$-coordinate of the prototype detector, see figure 1 , is determined by the propagation time of the scintillation light within each scintillator. We are measuring the time difference between the trigger signal and the light-response for those SiPMs, which are located at opposite ends of the detector module and therefore combined with different trapezoids. The position resolution is determined by the following equation.

$$
\text { position prediction in } x=\frac{1}{2} \cdot\left(t_{1}-t_{2}\right) \cdot \frac{c}{n}, \text { where }\left\{\begin{array}{l}
t_{1}=\text { time of flight in trapezoid } 1 \\
t_{2}=\text { time of flight in trapezoid } 2
\end{array}\right.
$$




\section{Prototype test at CERN}

In November 2012, the prototype module was tested in the H6 beam line at CERN with a $120 \mathrm{GeV} \pi$-beam. The detector halves were equipped with five WLS-fibers and five SiPMs [2], see figure 1. This corresponds to one sided readout of each trapezoid. All SiPMs were characterized via their breakdown voltage for equal gain conditions. The detector, including the SiPMs, were mounted in a temperature monitored light tight box, see figure 2 .

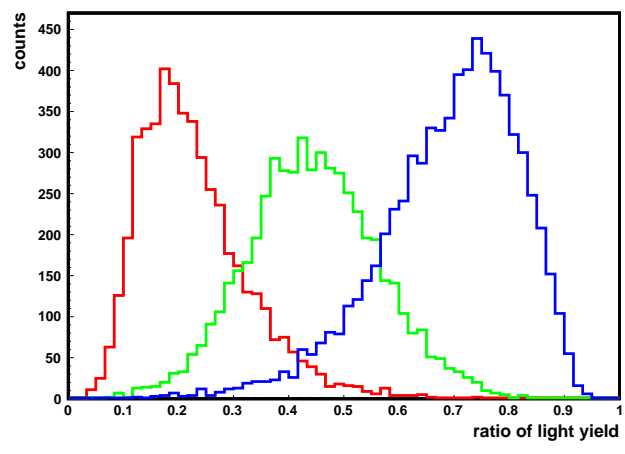

Figure 3: Spatial resolution in y-coordinate

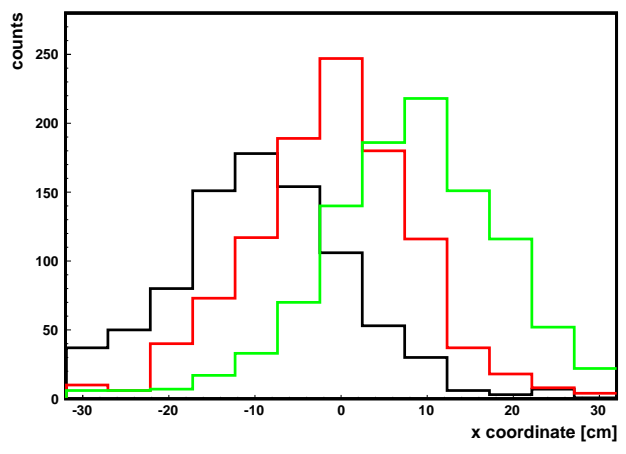

Figure 4: Spatial resolution in X-coordinate [3].

A coincidence trigger with an active area of $5 \mathrm{~mm}^{2}$, was used to start the data acquisition. The prototype detector was moved relatively to the beam and to the trigger to investigate the position resolution properties in $\mathrm{x}$ - and $\mathrm{y}$-direction. This is indicated by the colored dots in figure 2 and this coding is adopted for figure 3 and 4. SiPM-signals were recorded via VME-readout of a Flash-ADC-device [4].

\section{Results}

Within this beam time, a proof of principle was made to determine a spatial resolution of minimum ionizing particles due to two combined trapezoidal scintillators. We achieved a spatial resolution of $30 \mathrm{~mm}$ (FWHM), using the light yield ratio method, see figure 3. For the position resolution along the $\mathrm{x}$-coordinate, which results from the propagation time of light within the scintillator, we achieved a resolution of $10 \mathrm{~cm}$, which was limited by the readout electronics and corresponds to the expectation. Ongoing studies focuses on light propagation within the trapezoid and the enhancement of the detected light, where we gain a factor of two by using diffuse reflecting cover material. In combination with the usage of a more precise time measurement (factor of ten) the spatial resolution will be improved significantly in both directions in space.

\section{References}

[1] Saint-Gobain: Saint-Gobain, plastic scintillators, www.detectors.saint-gobain.com/ 10.10.2013

[2] Hamamatsu: SiPM S10362-11-050C, www.hamamatsu.com/ 10.10.2013

[3] Müller, R. : Development of a Position Sensitive Scintillating Detector, Master Th. LMU (2013)

[4] CAEN V1729: FADC Data Sheet, www.caen.it/ 10.10.2013 